\title{
La formation médicale continue en France : le bout du tunnel?
}

a Formation M édicale Continue (FMC) a pour objectif d'adapter les pratiques des médecins aux données scientifiques vali dées et aux progrès technologiques et thérapeutiques afin de proposer aux malades les meilleurs soins aux meilleurs coûts. Cet objectif vise à maintenir ou à augmenter la compétence des médecins et, par conséquent, leur expertise professionnelle.

En France, depuis des décennies, le FMC est un enjeu de pouvoirs et les débats passionnés des der nières années illustrent l'âpreté des discussions qui se sont développées à propos de la recherche du leadership sur son organisation et sur le contrôle de son financement. M algré ces difficultés, de nom breuses associations de FM C existent et fonction nent sur un mode souvent artisanal. Ceci n'a pas empêché, pour nombre d'entre elles, de mettre en cauvre des méthodes pertinentes de formation. Elles ont des moyens propres insignifiants, compensés, le plus souvent, par le soutien de l'Industrie phar maceutique. La FMC en France ne fait I'objet d'aucun contrôle objectif de qualité et les médecins après la sortie del'U niversiténe voient jamaisleur compétence réévaluée. Lesfinancements officiels de la FMC sont limités et ciblés; I'obtention des fonds nécessite des procédures administratives lourdes et décourageantes. II en résulte que les bénéficiaires sont le plus souvent des initiés profes sionnels ou semi-professionnels de la FM C. Enfin, le Conseil $\mathrm{N}$ ational de l'O rdre des médecins ou ses filiales régionales n'ont jamais pu jouer un rôle réel dans l'organisation de la FMC ou sur le contrôle du maintien de la compétence des médecins.

La parution récente de nouveaux textes portant sur l'organisation de la FM C en France suggère que nous nous trouvons aujourd'hui à la croisée des chemins. La perspective d'une F M C organisée, financée, et contrôlée semble réaliste. Toutefois, les expériences passées doivent inciter à la prudence car les mêmes causes produi sant les mêmes effets, il n'est pas exclu que de nouvelles difficultés pui ssent apparaître lors de la recherche d'un équilibre entre les partenaires potentiels ou lors du choix des méthodes de contrôle et des contrôleurs.

II est intéressant de regarder de l'autre côté de I'Atlantique et de sinspirer de la combinaison du professionnalisme et du pragmatisme de nos confrères québécois. La lecture de l'article de André Jacques et al. ${ }^{1}$ est instructive. Cet article rappelle le rôle majeur que joue l'Ordre des M édecins du Q uébec pour définir les secteurs rela tifs à l'exercice des médecinsqui doivent fairel'ob jet d'une surveillance. Cet article précise que les critères de qualité d'une activité de FMC sont définis par le Conseil de l'Education M édicale Continue du $Q$ uébec et que certaines associations professionnelles déterminent les exi gences en terme d'horaires de FM C pour leurs adhérents Enfin, le Collège des $M$ édecins du Q uébec, en tant qu'ordre professionnel a le mandat de veiller à l'existence d'une FM C dequalité pour les médecins et surtout il a pour rôle spécifique de contrôler la qualité des médecins en exercice.

Peut-on reproduire le modèle québécois en France? Pourquoi pas? M aisà condition quel'or ganisation, le financement et les contrôles se situent à l'échelle des régions. Ceci pourrait être le rôle de Comités Régionaux de Formation $M$ édicale Continue qui avaient été mis en place puis condamnés avant d'avoir fonctionné La composition et les fonctions spécifiques de ces comi tés régionaux restent à définir à la lumière des nouveaux textes mais leur réhabilitation est une urgente nécessité. Faute de quoi nous risquons de voir réapparaître une "usine à gaz » nationale qui a peu de chance d'être efficace.

Raymond COLIN raymond.colin@chu-rouen.fr

1.Jacques $A$, Goulet $F$, Leboeuf $S$. Lemaintien des compétences: un enjeu professionnel. Pédagogie M édicale $2002 ; 3: 116-121$. 